\title{
Transatlantica
}

Revue d'études américaines. American Studies Journal

$2 \mid 2013$

Jeux et enjeux du texte

\section{Jeux et enjeux du texte}

\section{René Alladaye et Sophie Vallas}

\section{(2) OpenEdition}

Journals

Édition électronique

URL : https://journals.openedition.org/transatlantica/6552

DOI : $10.4000 /$ transatlantica.6552

ISSN : 1765-2766

Éditeur

Association française d'Etudes Américaines (AFEA)

Édition imprimée

Date de publication : 31 décembre 2013

\section{Référence électronique}

René Alladaye et Sophie Vallas, « Jeux et enjeux du texte », Transatlantica [En ligne], 2 | 2013, mis en ligne le 16 mars 2022, consulté le 31 janvier 2023. URL : http://journals.openedition.org/ transatlantica/6552 ; DOI : https://doi.org/10.4000/transatlantica.6552

Ce document a été généré automatiquement le 31 janvier 2023.

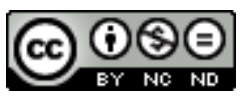

Creative Commons - Attribution - Pas d'Utilisation Commerciale - Pas de Modification 4.0 International - CC BY-NC-ND 4.0

https://creativecommons.org/licenses/by-nc-nd/4.0/ 


\title{
Jeux et enjeux du texte
}

\author{
René Alladaye et Sophie Vallas
}

1 Dans la première partie de Pourquoi la fiction?, Jean-Marie Schaeffer précise le cadre dans lequel il envisage son sujet, cadre «qui repose sur la conviction que la fiction artistique est une forme institutionnellement marquée et culturellement "évoluée" d'un ensemble de pratiques dont les exemplifications les plus fondamentales font partie intégrante de la vie de tous les jours (activités projectives, jeux fictionnels, jeux de rôles, rêves, rêveries, imaginations, etc)» (Schaeffer, 1999, 63). S'éloignant d'un débat fréquemment nourri autour de l'absence de valeur référentielle dans la fiction, il utilise au contraire nombre de récentes recherches scientifiques dans divers domaines pour étudier la façon dont "la combinaison d'un ensemble d'aptitudes cognitives, d'attitudes mentales ou d'activités psychiques plus élémentaires » (id.) permet à l'être humain de rêver, d'incarner un personnage, de jouer, d'inventer une histoire ou d'en écrire une. Schaeffer invite ainsi à

abandonner l'idée selon laquelle il existerait deux modalités de représentation, l'une qui serait fictionnelle et l'autre qui serait référentielle : il n'en existe qu'une seule, à savoir la modalité référentielle, ceci parce que la capacité représentationnelle est une structure neurologique qui a été façonnée de telle sorte par la sélection naturelle qu'elle fonctionne comme interface entre notre système nerveux central d'un côté, l'environnement extérieur ainsi que nos propres états et actes corporels et mentaux de l'autre. Donc, même si elle vise un objet inexistant, elle ne peut pas le représenter comme inexistant, parce que (se) représenter quelque chose revient à poser cette chose comme contenu représentationnel. Par ailleurs, les représentations fictionnelles posent exactement les mêmes classes de référents que ceux de la représentation commune : environnement extérieur, états et actes corporels et mentaux. Et cela vaut pour toutes les représentations, indépendamment de leur source, de leur mode d'accès ou de leur mode d'existence. Ainsi, quelle que soit la différence logique (du point de vue dénotationnel) entre la représentation d'un cheval et une représentation-de-licorne, elles sont équivalentes du point de vue du contenu. (Schaeffer, 1999, 153-54)

2 La représentation fictionnelle repose donc sur les mêmes mécanismes que ceux qui nous font nous représenter le monde autour de nous, mettant en jeu les mêmes 
activités neurologiques, corporelles et mentales. La production d'une fiction est une activité naturelle qui se développe dès la toute petite enfance, poursuit Schaeffer en reprenant les travaux de Winnicott ${ }^{1}$ : le bébé puis l'enfant et ses parents jouent sur ce terrain de jeux qu'ils partagent, à mi chemin entre fantasmes et réalité ; au jeu de l'enfant vient répondre le contre-jeu de l'adulte qui permet à l'enfant, dans ce que Schaeffer appelle "un accord de feintise partagée » (178), de reconnaître son jeu comme un jeu. La fiction, affirme-t-il ainsi, "naît comme espace de jeu, c'est-à-dire qu'elle naît dans cette portion très particulière de la réalité où les règles de la réalité sont suspendues»(175-76). L'enfant, remarque alors Schaeffer, commence par construire ses propres fictions (" autogènes ») avant de pouvoir « maîtriser des mondes fictionnels allogènes - c'est à dire des œuvres de fiction" (178). C'est donc le jeu originel avec l'entourage parental complice qui permet ultérieurement le développement de la fiction, définie d'ailleurs par Schaeffer comme une "feintise ludique partagée » - la fiction retient le « comme si », le " pour de faux » de la feintise sans toutefois chercher à tromper quiconque puisqu'elle repose sur un jeu librement engagé avec le lecteur in fabula. Née du jeu, la fiction est également un enjeu puisqu'elle est tout simplement essentielle à la construction d'un être humain.

3 Comment l'écrivain bâtit-il l'univers fictionnel dans lequel le lecteur viendra jouer et trouver le plaisir du texte? Le plaisir du jeu, tout d'abord, peut parfois donner l'impression de se situer à un niveau thématique évident, l'auteur entraînant son lecteur dans une rebondissante enquête criminelle (whodunits, mysteries et autres romans de détection), dans une patiente entreprise de décodage (romans d'espionnage) ou encore dans le déroulement d'un match mythique (baseball novels, par exemple). Mais cette transformation de la page blanche en un vert terrain de sport ou en une carte complexe sur laquelle se déroule une partie de chasse n'est souvent qu'un moyen pour l'auteur de rendre visible le jeu au cœur même de l'activité d'écriture. Dans l'article qui ouvre ce dossier, Noëlle Batt évoque dans un premier temps le versant «technique » du travail de l'artiste-artisan: l'auteur joue de toutes les stratégies et procédés que la narratologie a, depuis quelques décennies, analysés, catalogués, et sous ses mains expertes, le texte joue, lui aussi, comme on dit que le bois joue. D'où les aspérités du texte, ces moments, dit joliment Noëlle Batt, où « la lecture facile et fluide est soudain empêchée et oblige le lecteur qui trébuche à s'arrêter ». Vito Acconci, cité par Monica Manolescu dans son article, ne dit rien d'autre lorsqu'il proclame ainsi son amour pour l'écriture de Faulkner: "There's always a parenthesis, there's always something that stops that sentence from going to its goal that is a period». Une parenthèse, un mot qui fait saillie, une ellipse qui vous tourmente, une voix narrative fuyante, un cliché trop évident pour être honnête, une bifurcation syntaxique, une allitération obsédante, un adjectif improbable, une apposition presque insoutenable... Les possibilités sont innombrables de faire de la page un terrain de jeu où le lecteur est régulièrement déstabilisé par une langue qui ne lui est plus si familière que cela.

4 La métafiction a instauré le jeu comme ressort premier de l'écriture elle-même, et les romans métafictionnels ont rivalisé de virtuosité pour mettre à nu leurs propres mécanismes de production. Dans leurs infinis effets de miroirs, l'auteur devient bien souvent l'un des pions privilégiés. À une question sur le sens de la présence d'un personnage appelé Paul Auster au centre de son premier roman (City of Glass), Auster répond ainsi simplement : "I wanted to open up the process, to break down walls, to expose the plumbing " $(1989-90,555)$. La double présence de ce nom propre, sur la 
couverture du roman et dans ses pages, pour désigner deux écrivains identiques et différents à la fois, remet en question les catégories traditionnelles de l'auteur, du narrateur et du personnage et exhibe le texte comme une construction artificielle dans laquelle fictionnel et référentiel ne sont pas toujours aisément discernables. Un exercice en "plomberie apparente", somme toute, qui instaure l'auteur comme un joueur se choisissant lui-même comme enjeu de l'écriture fictionnelle. L'autofiction, cette écriture qui consiste pour un auteur à faire de soi une fiction, à écrire la fiction de soi, joue ainsi du nom propre, et c'est même, comme le rappelle Vincent Colonna, ce qui la caractérise en premier lieu. Vladimir Nabokov, Philip Roth ou Jerome Charyn, par exemple, ont tous, comme Auster et dans des textes fort différents les uns des autres, inséré leur nom sous de multiples combinaisons possibles (anagrammes, initiales, pseudonymes, etc.) dans leurs fictions, multipliant ainsi les identités, les masques et les reflets de leurs propres visages. Dans un texte malicieux publié peu de temps après Roland Barthes par Roland Barthes et intitulé avec esprit "Barthes puissance trois", Barthes ne remarque-t-il pas que "son propre nom, son Nom Propre» est "le plus éprouvé des pseudonymes " (Barthes, 1975b) ? Répondant aux questions de Philippe Forest au sujet de sa pratique de l'autofiction dans Le Roman vécu, Alain Jouffroy évoque le plaisir qu'il a pris à récupérer son " "temps retrouvé" à [lui]» (dans Forest et Gaugain, 2001, 485) dans ce roman : «C'est la fiction qu'un individu libre s'invente tout seul, en solitaire, à partir de matériaux disparates, incomplets, capricieux, lacunaires, de sa propre mémoire " (484), explique-t-il, avant de préciser : "Je me libère dans ce livre de ma propre biographie, je joue avec elle comme le chat avec la souris, je l'interprète comme une partition musicale » (485). Chat qui joue avec sa proie, musicien qui joue son morceau - l'enjeu n'est rien moins que la réinvention de l'auteur et peutêtre même l'invention de l'écrivain lui-même, celui qui, dans les termes de Marie Darrieussecq, s'amuse à «donner un équivalent-texte» de la vie (Darrieussecq, 2010, 286).

5 Les jeux du texte se cristallisent également aussi autour d'enjeux de réception. L'émergence de la littérature dite "postmoderne » a été marquée, pour en rester aux constats les plus simples, par une subversion affirmée des codes du récit « classique » et par un goût prononcé pour les formes ouvertes. Cette révolution s'est accompagnée d'une évolution cruciale de la théorie littéraire qui, à peu près au même moment, tendait à récuser l'autorité suprême de l'auteur pour accorder un rôle plus important - et même progressivement le rôle le plus important - au lecteur. Cette rupture avec une critique plus ancienne qui voyait dans l'auteur le dépositaire ultime du sens du texte fut inaugurée par le désormais célèbre article de Roland Barthes intitulé « la mort de l'auteur » dont les dernières lignes marquent un tournant spectaculaire : « Nous savons que, pour rendre l'écriture à son avenir, il faut en renverser le mythe : la naissance du lecteur doit se payer de La mort de l'auteur » (Barthes, 1984, 69; nous soulignons).

On a depuis fortement nuancé cette idée de la mort de l'auteur en montrant notamment que Barthes commet dans cet article une bévue théorique en confondant l'auteur au sens « concret » du terme (dont on peut effectivement décider de négliger la biographie et les opinions) et la notion - abstraite - d'auteur, au sens d'origine que tout texte suppose ${ }^{2}$. Cependant, force est de constater que cet article a ouvert une voie essentielle et contribué à redéfinir les enjeux du texte en posant pour la première fois l'idée que la littérature supposait une interaction entre le texte émis et celui qui le 
reçoit, en postulant donc, en d'autres termes, que le texte littéraire ne s'accomplit pleinement que lorsque le lecteur commence à jouer avec lui.

7 C'est cette idée d'un jeu mettant aux prises le texte et le lecteur, et obligeant ce dernier à quitter la posture du consommateur passif pour se transformer en acteur à part entière de l'échange littéraire que Barthes développe dans $S / Z$, notamment lorsqu'il introduit la notion de "scriptible $»^{3}$. Cette démarche prend appui, comme on le sait, sur une lecture linéaire et "codée » (au sens où elle repose sur cinq codes que Barthes définit comme outils de lecture) de la nouvelle de Balzac intitulée "Sarrasine», et culmine dans une analyse où le critique met en évidence dans le texte la présence d'une voix qui n'est autre que celle du lecteur :

La Zambinella, comme frappée de terreur, resta pensive (364).

Comme frappée de terreur : qui parle ici ? Ce ne peut être, même indirectement, Sarrasine, puisqu'il prend la crainte de la Zambinella pour de la pudeur. Ce ne peut être nommément le narrateur, puisqu'il sait, lui, que la Zambinella est effectivement terrifiée. La modalisation (comme) exprime les intérêts d'un seul personnage, qui n'est ni Sarrasine ni le narrateur et qui est le lecteur : c'est lui qui a intérêt à ce que la vérité soit à la fois nommée et esquivée, équivoque dont s'acquitte très bien le comme du discours, puisqu'il indexe la vérité et cependant la réduit déclarativement à une simple apparence. Ce que l'on entend ici est donc la voix déplacée que le lecteur prête, par procuration, au discours : le discours parle selon les intérêts du lecteur. Par quoi l'on voit que l'écriture n'est pas la communication d'un message qui partirait de l'auteur et irait au lecteur; elle est spécifiquement la voix même de la lecture : dans le texte seul parle le lecteur. (Barthes, 1970, 144-45)

8 La conclusion de l'analyse est sans doute quelque peu excessive, mais elle annonce l'émergence de la théorie de la réception et de travaux tels que ceux d'Umberto Eco qui met aussi l'accent sur l'importance de la participation du lecteur dans le processus interprétatif et sur la nécessité pour lui d'apporter sa pierre à l'édifice textuel :

Le texte est donc un tissu d'espaces blancs, d'interstices à remplir, et celui qui l'a émis prévoyait qu'ils seraient remplis et les a laissés en blanc pour deux raisons. D'abord parce qu'un texte est un mécanisme paresseux (ou économique) qui vit sur la plusvalue de sens qui y est introduite par le destinataire [...]. Ensuite parce que, au fur et à mesure qu'il passe de la fonction didactique à la fonction esthétique, un texte veut laisser au lecteur l'initiative interprétative, même si en général il désire être interprété avec une marge suffisante d'univocité. Un texte veut que quelqu'un l'aide à fonctionner. (Eco, 1979, 63-64)

9 Ce passage n'est pas sans évoquer la remarque de Michel Picard, qui souligne dans La lecture comme jeu, que la marque de tout texte, et plus particulièrement du texte de fiction, est d'avoir « du jeu » et que le propre de toute lecture est de "le faire jouer " (Picard, 1986, 48) ; l'ensemble nous ramène ainsi à Barthes, et à la formulation de ce qu'il appelle le " paradoxe du lecteur » :

Cette imagination d'un lecteur total - c'est-à-dire totalement multiple, paragrammatique - a peut-être ceci d'utile qu'elle permet d'entrevoir ce que l'on pourrait appeler le Paradoxe du lecteur : il est communément admis que lire, c'est décoder : des lettres, des mots, des sens, des structures, et cela est incontestable ; mais en accumulant les décodages, puisque la lecture est de droit infinie, en ôtant le cran d'arrêt du sens, en mettant la lecture en roue libre (ce qui est sa vocation structurelle) 
le lecteur est pris dans un renversement dialectique : finalement, il ne décode pas, il sur-code : il ne déchiffre pas, il produit, il entasse des langages, il se laisse infiniment et inlassablement traverser par eux : il est cette traversée. (Barthes, 1984, 47)

Plus récemment, Pierre Bayard a effectué un pas supplémentaire dans cette direction en fondant la notion de «critique policière ». L'idée, née d'une relecture particulièrement audacieuse de The Murder of Roger Ackroyd, le célèbre roman d'Agatha Christie, consiste à montrer que le lecteur peut remettre en question les faits posés par le récit lui-même, en l'occurrence la solution apportée au terme d'une enquête policière. Qui a tué Roger Ackroyd ?4 et L'Affaire du chien des Baskerville ${ }^{5}$ constituent deux exemples de ce type d'analyse ${ }^{6}$. Bayard invite son lecteur à reprendre pas à pas les enquêtes d'Hercule Poirot et de Sherlock Holmes pour mettre en évidence leurs oublis et incohérences et parvenir au constat que les deux détectives désignent à la fin de chaque livre le mauvais coupable, constat qui ouvre l'ultime étape dans laquelle il parvient à la véritable clé de l'énigme et dévoile l'identité du meurtrier'. La démarche de Bayard permet d'appréhender véritablement le texte dans sa dimension ludique et interactive. L'enjeu majeur que constitue la réception du texte littéraire se décrypte donc comme un jeu. La question se pose cependant de définir la nature exacte de ce jeu.

Pour ce faire, le plus simple est sans doute de partir de la définition de Johan Huizinga dans son ouvrage fondateur : Homo Ludens. Pour lui, le jeu est :

une action libre, sentie comme « fictive » et située en dehors de la vie courante, capable néanmoins d'absorber totalement le joueur ; une action dénuée de tout intérêt matériel et de toute utilité ; qui s'accomplit dans un temps et dans un espace expressément circonscrits, se déroule avec ordre selon des règles données et suscite dans la vie des relations de groupes s'entourant volontiers de mystère. (Huizinga, 1938, 35)

Dans sa classification, qui trouve son origine dans la définition de Huizinga, Roger Caillois classe les jeux en quatre catégories: les jeux fondés sur la lutte, le combat singulier, appartiennent au groupe "Agôn »; les jeux reposant sur le hasard (la loterie par exemple) se rattachent au groupe "Aléa »; les jeux fondés sur le simulacre et l'illusion constituent la catégorie "Mimicry"; enfin, les jeux ayant pour principe premier la transe, le vertige ou l'étourdissement, forment le groupe " Ilinx $»^{8}$.

Cette classification peut-elle nous aider à mieux saisir les enjeux de la réception du texte littéraire ? Ilinx semble absent du champ littéraire, et Alea ne s'applique qu'à des cas très particuliers. La lecture des Cent mille milliards de poèmes de Queneau, ou, pour être plus précis, l'exercice de « composition en second » que ce livre suppose de la part $\mathrm{du}$ lecteur, pour prendre un exemple emblématique, peut tout à fait s'effectuer de façon aléatoire. Le livre se compose de dix feuilles, chacune séparée en quatorze bandes horizontales, chaque bande portant sur son recto un vers. Le lecteur peut donc, en tournant les bandes horizontales comme des pages, choisir pour chaque vers une des dix versions proposées par Queneau. Les dix versions de chaque vers ont la même scansion et la même rime, ce qui assure que chaque sonnet ainsi assemblé est régulier dans sa forme. Il y a donc $10^{14}$ poèmes potentiels. Cependant, si la lecture peut s'effectuer sans schéma particulier et en quelque sorte " au hasard », il est clair que la composition du livre répond, pour sa part, à un ensemble de règles extrêmement strictes, garantes de son fonctionnement ${ }^{9}$. L'éventualité d'une réception aléatoire est donc préparée par une composition extrêmement concertée et stricte. amener à jeter un pont entre la lecture littéraire et le groupe des jeux sur lequel Caillois 
appose l'étiquette « mimicry ». Les nombreux clubs et sociétés honorant par exemple la mémoire de Sherlock Holmes et organisant régulièrement des rencontres au cours desquelles les membres arborent la tenue de leur détective favori témoignent de ce lien entre la littérature et une activité qui se rapproche très fortement du jeu de rôles.

Cette forme d'imitation amène du reste à envisager son pendant abstrait, la question de l'identification. Comme le montre bien la notion de " willing suspension of disbelief ", c'est le propre du discours fictionnel que de s'adosser à un «faire semblant », l'acte ludique par excellence (notamment chez l'enfant). Ce «faire semblant» prend fréquemment chez le lecteur récréatif la forme de l'identification, cette "métempsycose provisoire durant laquelle le lecteur se [met] dans la peau d'un personnage, se [laisse] habiter par lui ", " cette possession éphémère et bénigne » (Picard, 1986, 91). L'identification n’a pas nécessairement bonne presse. Elle est proscrite dans le contexte des études littéraires parce qu'elle implique que l'on succombe à l'illusion référentielle (Nabokov la condamne d'entrée de jeu dans ses cours de littérature) ${ }^{10}$. Picard, cependant, en offre une vision plus nuancée, notamment en soulignant la complexité du concept d'identification dont la portée dépasse le simple champ des études littéraires ${ }^{11}$.

Le groupe que Caillois nomme "Agôn » est celui qui entretient manifestement le plus de liens avec l'échange littéraire. Il est en effet possible de concevoir ce dernier comme une sorte de compétition, une lutte entre le texte et son lecteur, ou plus exactement une lutte entre le lecteur et l'auteur (au sens d'intentionnalité) dont le texte est le vecteur. Si la théorie de la réception a montré que le lecteur jouissait d'une initiative importante dans la construction du sens du texte littéraire, la question se pose en effet de savoir qui dicte cette réception. En d'autres termes, le lecteur est-il libre face au texte, ou le travail de "sur-codage » qu'évoque Barthes se fait-il selon des modalités prévues en dehors de lui et qui lui échappent? Intervient ici le problème du possible divorce entre l'intentionnalité de l'auteur et celle du lecteur. Eco formule, dans Lector in fabula, ce qui peut apparaître comme une synthèse du problème. En effet, l'accent mis sur le fait que le texte a besoin du lecteur pour fonctionner se double d'un corollaire important: l'auteur, en le composant, a anticipé les manières dont le lecteur serait susceptible de le recevoir, et donc de le faire fonctionner :

Nous avons dit que le texte postule la coopération du lecteur comme condition d'actualisation. Nous pouvons dire cela d'une façon plus précise : un texte est un produit dont le sort interprétatif doit faire partie de son mécanisme génératif; générer un texte signifie mettre en œuvre une stratégie dont font partie les prévisions des mouvements de l'autre - comme dans toute stratégie. Dans la stratégie militaire (ou dans celle des échecs, disons dans toute stratégie de jeu), le stratège se dessine un modèle d'adversaire. [...] Cependant, dans la stratégie militaire (à la différence de celle des échecs), des impondérables peuvent intervenir. [...] Tout bon stratège doit donc tenir compte de ces événements fortuits, par un calcul de probabilités. Or il en va de même pour les textes. L'auteur du texte devra donc agir de façon identique : « Le bras du lac de Côme qui s'étend vers le sud... » : et si je tombe sur un lecteur qui n'a jamais entendu parler du lac de Côme ? Je dois faire en sorte de le récupérer plus loin, pour le moment faisons comme si Côme était un flatus vocis, comme Xanadou. Ensuite je ferai des allusions au ciel de Lombardie, au rapport entre Côme et Milan et Bergame, à la situation de la péninsule italienne. Bref, le lecteur qui présente une carence encyclopédique est attendu tôt ou tard au tournant. (Eco, 1979, 65-66) 
Une fois le texte publié, le lecteur a l'initiative, mais l'auteur a calculé plusieurs coups à l'avance et prévu une bonne partie des réponses dudit lecteur, voire, comme le montre Eco, son absence de réponse. La liberté du lecteur est donc toujours étroitement encadrée, surveillée. L'allusion d'Eco au jeu d'échecs nous mène vers Nabokov qui pose une idée similaire dans un passage de Speak, Memory:

I remember one particular problem I had been trying to compose for months. There came a night when I managed at last to express that particular theme. It was meant for the delectation of the very expert solver. The unsophisticated might miss the point of the problem entirely, and discover its fairly simple, «thetic " solution without having passed through the pleasurable torments prepared for the sophisticated one. The latter would start by falling for an illusory pattern of play based on a fashionable avant-garde theme (exposing White's King to checks), which the composer had taken the greatest pains to " plant » (with only one obscure little move by an inconspicuous pawn to upset it). Having passed through this « antithetic » inferno the by now ultrasophisticated solver would reach the simple key move (Bishop to c2) as somebody on a wild goose chase might go from Albany to New York by way of Vancouver, Eurasia and the Azores. The pleasant experience of the roundabout route (strange landscapes, gongs, tigers, exotic customs, the thrice-repeated circuit of a newly married couple round the sacred fire of an earthen brazier) would amply reward him for the misery of the deceit, and after that, his arrival at the simple key move would provide him with a synthesis of poignant artistic delight. (Nabokov, 1967, 291-92)

Ce passage peut bien sûr être lu au premier degré : Nabokov, on le sait, composait des problèmes d'échecs à ses heures perdues ${ }^{12}$. Cette citation décrit donc les étapes par lesquelles devra passer l'amateur se confrontant au problème décrit ici et tentant de le résoudre ${ }^{13}$. Le passage décrit la manière dont il va envisager plusieurs éventualités et finalement parvenir à la seule qui permette de donner mat en deux coups. Mais comme l'a montré Chris Ackerley ${ }^{14}$, ces lignes peuvent aussi être lues au second degré, comme une allégorie du jeu de la réception d'un texte littéraire. À l'image d'un problème d'échecs, un texte est un écheveau à démêler, une combinatoire à décrypter. L'auteur est l'équivalent du compositeur du problème et le lecteur de l'amateur tentant d'en trouver la clé. Et Nabokov de décrire dans "Good Readers and Good Writers", l'introduction au premier volume de ses cours de littérature, ce moment de grâce qu'est la rencontre de l'auteur et de son lecteur au sommet de la montagne (l'œuvre, on l'a deviné) que ce dernier vient de gravir en ayant franchi tous les obstacles: «Up a trackless slope climbs the master artist, and at the top, on a windy ridge, who do you think he meets? The panting and happy reader, and they spontaneously embrace and are linked forever if the book lasts forever » (Nabokov, 1983, 2).

Nabokov entretient une vision très hiérarchisée et manichéenne des lecteurs. Il y a pour lui, les bons et les mauvais, et puis, bien loin au-dessus, le meilleur d'entre tous, lui-même ${ }^{15}$. Que signifie être un « bon lecteur » ? Voir beaucoup de choses dans le texte, y compris des détails a priori insignifiants, développer une perception intime de ce qui s'y passe et être ainsi en mesure d'en apprécier l'originalité, l'harmonie, la complexité et l'élément de tromperie inhérent à toute production artistique, cette splendide insincérité qu'évoque Nabokov dans un entretien où il explique la réunion, dans Poems and Problems, de poèmes et de problèmes d'échecs:

Why are you including the chess problems with the poems? 
Because problems are the poetry of chess. They demand from the composer the same virtues that characterize all worthwhile art: originality, invention, harmony, conciseness, complexity, and splendid insincerity. (Nabokov, 1973, 160-61)

Chaque page devient ainsi l'occasion de provoquer le lecteur d'une question : êtes-vous capable de détecter tout ce que j'ai mis dans mon texte? Ce dernier fonctionne comme une série d'énigmes que l'on pose et dont on donne la solution au détour d'une page, de manière directe ou plus oblique.

Le corollaire de cette analogie entre texte littéraire et problème d'échecs est simple : le lecteur, à son meilleur, ne peut que résoudre le problème posé par l'auteur, et dont ce dernier a calculé la solution dans les moindres détails, cartographiant le labyrinthe des variantes. La lecture devient un combat singulier entre le lecteur et l'auteur dans lequel ce dernier a, par définition, toujours le dernier mot. Car si le lecteur croit parfois s'être montré à la hauteur et semble avoir triomphé des complexités placées en travers de son chemin, il ne tarde pas à se rendre compte qu'il n'en est rien et qu'il s'est fait piéger. L'allusion, dans Lolita, à un poème célèbre de Goethe intitulé "Erlkönig " ("Le Roi des Aulnes »), est emblématique de ce genre de "mésaventure " ${ }^{16}$. Juste avant que Lolita soit « enlevée », Humbert, le narrateur, fait allusion à Quilty en mentionnant « a heterosexual Erlkönig in pursuit » (Nabokov, 1955, 273). Mais la satisfaction d'avoir vu la référence est sensiblement tempérée lorsque l'on prend conscience du fait que ce clin d'œil contenant le titre même du poème, constitue en fait la réponse à une énigme posée plus haut et que le lecteur était - déjà - censé repérer... Le poème de Goethe raconte, pour le résumer très rapidement, qu'une incarnation spectrale du Roi des Aulnes poursuit à travers la forêt un père et son enfant, et finit par tuer ce dernier dans la dernière strophe du poème : « Le père frissonne d'horreur, il galope à vive allure, / Il tient dans ses bras l'enfant gémissant, / Il arrive à grand-peine à son port ; / Dans ses bras l'enfant était mort $\aleph^{17}$. Si on connaît ce vers, on songera au moment où Humbert et Lolita sont sur le point d'arriver à l'hôtel Enchanted Hunters (Partie I, chapitre 27). Lolita suggère alors une sortie au cinéma, proposition à laquelle Humbert réagit de la façon suivante: "We might," chanted Humbert - knowing perfectly well, the sly tumescent devil, that by nine, when his show began, she would be dead in his arms" " (Nabokov, 1955, 131 ; c'est moi qui souligne).

On reconnaît le dénouement du poème de Goethe et on comprend, mais un peu tard, que c'était cela qu'on attendait du bon lecteur: qu'il repère la citation légèrement transformée de ce dernier vers avant de voir son intuition confirmée par l'allusion transparente qui se trouve cent quarante pages plus loin. On comprend aussi qu'à ce jeu-là, gagner contre Nabokov tient du génie ou du miracle.

L'un des enjeux de ce jeu de la réception est donc bien un enjeu de pouvoir, et la question celle de savoir qui a véritablement barre sur le texte. Une réponse semble s'imposer : le lecteur joue avec le texte, au sens où il lui est possible d'en renouveler la lecture, parfois même bien au-delà de ce que l'auteur pouvait envisager, cela est indéniable (Eco donne l'exemple caractéristique de la lecture freudienne de l'œdipe de Sophocle ${ }^{18}$ ). Mais on doit admettre que, le plus souvent, il joue ce texte comme un musicien joue une partition, ou comme un amateur d'échecs résout un problème, en accomplissant les mouvements et en respectant les inflexions prévus par le compositeur. On doit admettre, enfin, qu'il est parfois joué par le texte (ou que le texte se joue de lui) sans qu'il en ait toujours clairement conscience, et qu'il est bien difficile 
de s'affranchir de la tutelle de l'auteur, surtout quand ce dernier est aussi retors et calculateur que Nabokov.

À moins que cette question du jeu entre auteur, narrateur et lecteur et des enjeux de pouvoir qu'il entraîne ne soit finalement qu'un leurre. Si Jorge Luis Borges a raison, si «l'univers (que d'autres appellent la bibliothèque)» (Borges, 1957, 71) n'est que vaste fiction qui comprend tous les livres, si les livres sont des livres de sable au « nombre de pages [...] exactement infini » (Borges, 1975, 141), si le récit d'un narrateur peut être le rêve de son personnage au seuil de la mort, si l'écrivain produit sa propre hypothèse, est son propre personnage et n'est rien d'autre que ses lectures, si Pierre Ménard peut être l'auteur de Don Quichotte sans avoir jamais copié la moindre page de celui de Cervantès, alors auteur et lecteur sont interchangeables, fiction l'un et l'autre, et errent sans savoir lequel des deux écrira le prochain livre dont ils ne savent pas non plus quel autre livre il redira, ou de quel auteur ou lecteur il sera le rêve - soumis à cette loterie incarnée à "Babylone [qui] n'est autre chose qu'un infini jeu de hasards » (Borges, 1957, 69).

\section{Présentation du numéro}

Noëlle Batt ouvre ce dossier avec un texte sur la puissance du roman, qu'elle place à l'ombre de Proust. Comment écrit-on quand on écrit "parce qu'il y a urgence à dire ", parce qu'on n'est «bon qu'à ça ", comme disait Beckett ${ }^{19}$ ? Et comment le récit né de cette urgence en vient-il à révéler plus que le discours philosophique ? Noëlle Batt cite Vincent Descombes évoquant non pas «le langage » mais l'existence de «plusieurs jeux de langage », et nous permet ainsi d'entrer dans le vif du texte : là où le romancier, artiste mais " aussi artisan », joue de toutes les techniques fictionnelles et narratives de son outil, mais sait également entraîner le lecteur dans une expérience de lecture « non seulement active mais activante » qui le mènera à « l'effet esthétique ».

C'est une nouvelle de Paul Auster ("Auggie Wren's Christmas Story») que Linda Collinge s'amuse à lire, précisément, comme un conte de Noël, une histoire sur l'écriture et une leçon sur "la lecture comme jeu», comme si Auster avait voulu illustrer l'ouvrage de Michel Picard, remarque-t-elle. Dans ce petit texte qui brouille d'emblée les frontières entre fiction et non fiction, qui joue tout à la fois sur l'illusion référentielle et l'illusion littéraire, le jeu complice qui se développe entre Auggie et la vieille dame esseulée un soir de Noël reflète celui que le conteur établit avec son auditeur; le cadrage plus large de l'histoire, dans le viseur de l'appareil photographique d'Auggie, rappelle au narrateur comme au lecteur ce qui se joue dans le processus de réception et dans l'expérience esthétique.

Le lecteur dont se joue l'auteur - c'est bien ce que Marie Olivier se propose d'analyser également. À la fois exercice de réception et de composition, la traduction incarne par excellence les enjeux subtils des jeux textuels. Marie Olivier en explore les méandres dans une étude consacrée à la traduction par Louise Glück d'un poème de Pouchkine, traduction bien particulière cependant en ceci qu'elle refuse de s'avouer telle et se présente, dans le recueil intitulé Averno, comme un poème original, "Omens". Brouillage entre le russe et l'anglais, l'original et la copie, l'hypotexte et l'hypertexte, naissance du transtexte... Marie Olivier analyse en détail la subtile " démarche de jeu et de manipulation de la lettre » à l'œuvre à travers « la réécriture et l'appropriation d'un texte écrit dans une langue étrangère ». 

s'intéresse aux jeux vidéos, depuis leur apparition dans les années 1970 jusqu'à aujourd'hui. Quel est le potentiel narratif des jeux vidéos?, se demande-t-il en se proposant d'analyser leur dimension plus ou moins narrative en fonction des époques, des possibilités techniques offertes aux concepteurs mais aussi des tendances. Devant son écran, le joueur est à la fois spectateur, lecteur et acteur, et les jeux le confrontent à des phases d'action, qui sollicitent pleinement sa participation, et à des phases de narration dans lesquelles une histoire lui est proposée de façon plus ou moins dirigiste. Quelles sont les frontières entre jeu et narration? Jusqu'à quel point le jeu vidéo laisset-il le joueur jouer, et dans quel but déploie-t-il parfois tant d'activités pour l'en empêcher? également omniprésent puisque les personnages y pratiquent le baseball, le football, le tennis ou encore les échecs. Ces sports et ces jeux sont utilisés par Fountain comme autant de métaphores d'un vaste jeu géopolitique où les règles sont viciées, les dés pipés, les cartes truquées. Lorsque la propagande noyaute le langage, lorsque l'enrôlement pervertit les jeux de rôle, lorsque le double jeu rend toute règle caduque, certains personnages comprennent la nécessité de décoder les règles imposées, de les subvertir et de créer les leurs, de réactiver la fonction ludique du langage et d'écrire leurs propres fictions.

Fabrice Lyczba travaille sur tout un matériau épitextuel ludique accompagnant la sortie des films hollywoodiens des années 1920 (affiches, textes promotionnels et reportages journalistiques sur les tournages, pratiques commerciales, petits événements organisés par les exploitants de salles, etc.) et montre comment, à l'époque, on s'attache déjà à déconstruire le soi-disant réalisme du cinéma américain en exhibant l'envers du décor. Le spectateur fasciné pénètre au cœur même de l'usine à rêves et contemple la façon dont le cinéma met en jeu et en scène le réel. Le discours médiatique et promotionnel hollywoodien, dans le but de mieux expliquer comment on parvient à l'authentique, en vient donc "à révéler cet "authentique" comme un artifice de production » et crée ainsi une nouvelle forme de spectature.

31 C'est également l'envers $\mathrm{du}$ décor qui intéresse Céline Manresa, et plus particulièrement la façon dont les choses, dans A Lost Lady et My Mortal Enemy de Willa Cather, fonctionnent comme des paravents, des écrans, des leurres qui voilent et dévoilent les coulisses d'intérieurs soigneusement composés et, au-delà de ces espaces de représentation, les coulisses de toute une société américaine. Éléments essentiels des intrigues qu'elles font basculer, les choses sont partie intégrante des codes de conduite, participent pleinement aux jeux de séduction, lient les amants et attisent les désirs. Ce rôle si important des choses introduit un «jeu dans la progression des récits » dont Céline Manresa met en évidence les délicats rouages.

L'univers de Vito Acconci ne saurait être plus éloigné de celui de Willa Cather, et pourtant Monica Manolescu montre à quel point, chez cet artiste qui a d'abord cherché une écriture littéraire sur la page avant de sortir dans la marge de la rue où il a multiplié les performances, les mots sont des choses avec lesquelles il joue comme avec des briques de Lego. Pour Acconci, en effet, le langage ne s'écrit pas mais se construit, et toute sa recherche artistique a consisté à désapprendre règles et conventions afin d'ouvrir un tout nouvel espace de jeu et de se donner des contraintes dont le but premier est de désorienter tant l'artiste lui-même que le lecteur/spectateur. 
Yannicke Chupin, enfin, signe le dernier texte de ce dossier, dans lequel elle propose une étude de la dimension métafictionnelle de The Anthologist. Le héros du roman de Nicholson Baker est un écrivain dans l'impasse, tout aussi incapable de mettre en forme l'anthologie poétique sur laquelle il travaille que de rédiger son introduction théorique. Lorsque, seul dans un grenier, il s'invente un terrain de jeu, multiplie les rôles et les masques, hallucine les poètes du passé, dialogue avec des partenaires imaginaires et se rit du discours critique, il redécouvre la jouissance du jeu, les plaisirs du faux-semblant, la saveur de l'artifice, et retrouve le chemin de la poésie qu'il célèbre - enfin - de tout son corps et de toute sa voix.

ACKERLEY, Chris, « Three notes Towards a Thetic Solution », Nabokov Studies 2, 1995, 87-103.

ALLADAYE, René, «Play hard : enjeux des jeux dans Lolita », Miranda, n³, 2010, $<$ http://www.mirandaejournal.fr/1/miranda/article.xsp?

numero=3\&id_article=article_05-657>.

AUSTER, Paul, « Interview with Larry McCaffery and Sinda Gregory » (1989-90), reprinted in Collected Prose, New York, Picador, 2010.

BARTHES, Roland, Roland Barthes par Roland Barthes, Paris, Seuil, coll. «Écrivains de toujours », 1975.

---, « Barthes puissance trois », La Quinzaine littéraire, n²05, mars 1975.

---, S/Z (1970), Paris, Seuil, coll. « Points », 1976.

---, Le Bruissement de la langue, Paris, Seuil, coll. « Points », 1993 [1984].

BAYARD, Pierre, Qui a tué Roger Ackroyd ?, Paris, Éditions de Minuit, 1998.

---, Enquête sur Hamlet. Le Dialogue de sourds, Paris, Éditions de Minuit, 2002.

---, L'Affaire du chien des Baskerville, Paris, Éditions de Minuit, 2008.

BORGES, Jorge-Luis, Fictions, Paris, Gallimard, coll. Folio, 1986 [1957].

---, Le Livre de sable, Paris, Gallimard, coll. Folio, 1992 [1975].

CAILLOIS, Roger, Les Jeux et les hommes, le masque et le vertige, Paris, Gallimard, 1967

[1958].

COMPAGNON, Antoine, Le Démon de la théorie, Paris, Seuil, coll. « Points », 2001 [1998].

DARRIEUSSECQ, Marie, Rapport de police, Paris, P.O.L, 2010.

ECO, Umberto, Lector in fabula, Paris, Livre de Poche, coll. « Biblio Essais », 1989 [1979]. FOREST, Philippe et Claude GAUGAIN, Les Romans du je, Nantes, Éditions Pleins Feux, coll. « Horizons comparatistes », 2001.

HUIZINGA, Johan, Homo Ludens, essai sur la fonction sociale du jeu, Paris, Gallimard, 1976 [1938].

NABOKOV, Vladimir, Lolita, Harmondsworth, Penguin Red Classics, 2006 [1955].

---, Speak, Memory, an Autobiography Revisited, New York, Vintage International, 1989

[1967].

---, Poems and Problems, New York, MacGraw-Hill, 1971.

---, Lectures on Literature, Londres, Picador, 1983 [1980].

---, Strong Opinions, New York, Vintage International, 1990 [1973].

PICARD, Michel, La Lecture comme jeu, Paris, Éditions de Minuit, coll. «Critique », 1986. RIFFATERRE, Michael, «L'illusion référentielle », Littérature et réalité, Paris, Seuil, coll. « Points », 1982.

SCHAEFFER, Jean-Marie, Pourquoi la fiction?, Paris, Seuil, coll. « Poétique », 1999. 


\section{NOTES}

1. Notamment Jeu et réalité. L'Espace potentiel, Paris, Gallimard, 1975.

2. Voir Antoine Compagnon, 1998, 58.

3. «Pourquoi le scriptible est-il notre valeur? Parce que l'enjeu du travail littéraire (de la littérature comme travail), c'est de faire du lecteur, non plus un consommateur, mais un producteur du texte » (Barthes, 1970, 10).

4. Pierre Bayard, Qui a tué Roger Ackroyd ?, Paris, Éditions de Minuit, 1998.

5. Pierre Bayard, L'Affaire du chien des Baskerville, Paris, Éditions de Minuit, 2008.

6. On pourrait inclure dans cette liste Enquête sur Hamlet. Le Dialogue de sourds, Paris, Éditions de Minuit, 2002.

7. L'idée de voir dans le lecteur une figure de détective n'est d'ailleurs pas complètement nouvelle. Nabokov l'évoque dans l'épigraphe de Lectures on Literature: «My course, among other things, is a kind of detective investigation of the mystery of literary structures ».

8. Roger Caillois, Les jeux et les hommes, le masque et le vertige, Paris, Gallimard, 1967 [1958].

9. Pour n'évoquer qu'une de ces règles, les rimes des sonnets suivent le schéma $: a b$ a b / $a b$ a $b$ / c c d / e e d. Dans ce schéma, a, c et e sont des rimes féminines (respectivement en -ise, -otte et oques) tandis que $b$ et $d$ sont des rimes masculines (en [o] et -in).

10. Voir Nabokov, 1980, 3.

11. Voir Picard, 1986, 91-95.

12. On trouve d'ailleurs certaines de ses compositions dans un volume intitulé Poems and Problems, New York, MacGraw-Hill, 1971.

13. Il peut être utile de préciser ici ce qu'est un problème d'échecs. Il ne s'agit pas d'une vraie partie, mais d'une position composée qu'il faut résoudre. Le plus souvent, le but est de trouver une combinaison qui mène à un échec et mat en deux ou trois coups, et dont le premier mouvement, la clé du problème, est remarquable par son caractère inattendu, voire paradoxal. Plus la clé est surprenante, et les variantes liées à sa solution diverses et surprenantes, plus la valeur esthétique du problème est grande. À toutes fins utiles, voici la position de départ de celui dont il est ici question. Blancs : Ra7, Db6, Tf4, Th5, Fe4, Fh8, Cd8, Ce6, pions b7, g3. Noirs : Re5, Tg7, Fh6, Ce2, Cg5, pions c3, c6, d7. Les Blancs jouent et font mat en deux coups. Essayez d'oublier que vous avez lu la citation précédente...

14. Dans son étude intitulée "Three notes Towards a Thetic Solution ", (Nabokov Studies 2, 1995, 87-103), Chris Ackerley postule l'existence d'un lien étroit entre le problème d'échecs évoqué dans ce passage et la structure de Pale Fire.

15. "I don't think an artist should bother about his audience. His best audience is the person he sees in his shaving mirror every morning " (Strong Opinions, New York, Vintage International, 1990 [1973], 18).

16. Je reprends ici une analyse déjà publiée dans "Play hard : enjeux des jeux dans Lolita ", Miranda, $\mathrm{n}^{\circ} 3,2010$.

17. Je reprends ici la traduction française (pas toujours exempte de reproches) de Charles Nodier.

18. Voir Umberto Eco, 1979, 233-34.

19. Réponse donnée à la question « Pourquoi écrivez-vous? " posée par Libération dans un horssérie (mars 1985). 


\section{AUTEURS}

RENÉ ALLADAYE

Université Toulouse 2-Le Mirail

SOPHIE VALLAS

Université Aix-Marseille 